\title{
Design of Multivalent Ligands that Cross the Blood Brain Barrier for the Treatment of Neuropathic Pain Without Toxicities
}

\author{
Victor J. Hruby ${ }^{1}$, Takashi Yamamoto ${ }^{1}$, Aswini Kumar Giri', \\ Davis S. Herman ${ }^{2}$, Tally Largent-Milnes ${ }^{2}$, Todd Vanderah ${ }^{2}$, and Frank Porreca \\ ${ }^{1}$ Dept. of Chemistry and Biochemistry; ${ }^{2}$ Dept. of Pharmacology, University of Arizona, Tucson, AZ, 85721, USA
}

\section{Introduction}

Pain, especially prolonged and neuropathic, is the most ubiquitous and expensive disease in the U.S. and worldwide and treatments are inadequate or ineffective and often lead to enhanced pain sensitivity, addiction and poor quality of life. New modalities for treatment are urgently needed but are not forthcoming from pharma. We have proposed that a new approach to drug design is needed which takes into account the changes in the expressed genome that accompany these disease states [1]. In particular, in prolonged and neuropathic pain there is up-regulation of neurotransmitters and their receptors in ascending and descending pain pathways that are stimulatory and therefore enhance the pain perception. From these and other findings, we have hypothesized that the design of a multivalent ligand that has agonist activity at the mu and delta opioid receptors (balanced or favoring $\mu$ or $\delta$ receptors) with antagonist activities at the up-regulated stimulatory receptor (e.g. ligands for neurokinin-1 (NK-1) receptor) all in single ligand, would have potent analgesic activities but without the toxicities of current opioid drugs and without the development of tolerance or addiction.

\section{Results and Discussion}

The design of a multivalent ligand with multiple pharmacophores all in a single molecule requires special considerations in drug design. Each pharmacophore for each target requires a unique structure, and each receptor-binding pocket for each pharmacophore has a unique 3D structural requirement. Each unique pharmacophore must be in the designed ligand and they must not interfere with each other when they bind to their respective receptor/acceptor. Therefore, the issues of potential overlapping pharmacophores or separated pharmacophores by appropriate biocompatible linkers must be considered as part of the design process. Though we have had failures, we also have had several successes. Here we will discuss the design and biological properties of trivalent ligands that have mu and delta opioid receptor agonist activity and neurokinin-1 receptor antagonist activity, all in a relatively simple peptidomimetic scaffold that crosses the blood brain barrier.

Our specific design of multivalent ligands is quite simple because there is much that we and others in the literature have done in examining the SAR of mu and delta opioid agonists and NK-1 receptor antagonists. Among the key pharmacophore structural moieties for mu/delta agonists is a free amino terminal group, and the NK-1 receptor antagonists require a specific nonpeptide moiety at the $C$-terminal. Thus a linear peptide serves both pharmacophores well with an intervening linker or address moiety.

$$
\text { Mu/Delta Ag.--Spacer/Address-NK-1 R. Ant. }
$$

Fig. 1. Design of Mu-Delta Opioid Receptor Agonist, NK-1 Receptor Antagonist in a Multivalent Ligand.

In our design we used a linker amino acid that also serves as an address for all three receptors. In Table 1, we provide the structures of three of our lead peptides which serve as the basis for what led us to attain a ligand with all the desired biological properties and none of the toxicities or development of tolerance or addictive behaviors found in current opioid drugs. The results of binding at human delta 
Table 1. Structure and selected biological activities of trivalent ligands for treatment of pain.

\begin{tabular}{ccccc}
\hline Structure & $K_{i}$ & $K_{i}$ & $K_{i}$ & $K_{i}$ \\
& $(\mu)$ & $(\delta)$ & $h N K I$ & $n N K I$ \\
& $n M$ & $n M$ & $n M$ & $n M$ \\
\hline 1) Tyr-D-Ala-Gly-Phe-Met-Pro-Leu-Trp-3',5'-Bn $\left(\mathrm{OF}_{3}\right)_{2}$ & 36 & 2.8 & 0.080 & 0.29 \\
2) Tyr-D-Ala-Gly-Phe-Met-Pro-Leu-Trp-NH-3',5'-Bn $\left(\mathrm{OF}_{3}\right)_{2}$ & 16 & 0.66 & 0.006 & 7.3 \\
3) DMT-D-Ala-Gly-N-MePhe-Pro-Leu-Trp-NH-3',5'-Bn $\left(\mathrm{OF}_{3}\right)_{2}$ & 0.71 & 6 & 2.2 & 4.8 \\
\hline
\end{tabular}

and mu receptors and at human and rat neurokinin receptors are provided. Note that compounds and $\mathbf{1}$ and $\mathbf{2}$ are delta opioid receptor selective, while $\mathbf{3}$ is mu opioid receptor structure selective.

We also have examined second messenger cAMP activities and efficacies, as well as MVD and GP1 tissue assay activities (for $\mathbf{1}$ and $\mathbf{2}$ see references [2] and [3] for data). In all cases, the compounds are agonists at opioid receptors and antagonists at neurokinin 1 receptors (for $\mathbf{3}$, manuscript in preparation). Extensive in vivo_studies of analgesic activity in acute and neuropathic pain models have been made. To summarize, our 3 compounds show potent analgesic effects in both acute and neuropathic pain models given i.p. or given i.v., and all three compounds cross the blood brain barrier. In addition, we have demonstrated that these ligands have none of the toxicities associated with morphine long term use including: 1) no motor impairment; 2) no development of tolerance after several days of administration of drug; 3) no development of place preference, an assay for addiction potential after several days of administration; and 4) no inhibition of gut transit (constipation) as well as others $[3,4,5]$.

We are very excited about these findings which strongly suggest that this multivalent approach to drug has great potential for the development of novel drugs for the treatment of prolonged pain without toxicities or the development of tolerance, and our approach can find applications to other degenerative diseases.

\section{Acknowledgments}

We thank Christine Hiner Kasten for helping in preparing the manuscript. Support by grants from the U.S. Public Health Service, Natural Institute of Health, NIDA, POI DA 006284 and NIDA R01 DA 13449.

\section{References}

1. Hruby, V.J., et.al. Am. Assoc. Pharm-Sci. J. 8, E450-460 (2000), http://dx.doi.org/10.1208/aapsi080353

2. Yamamoto, T. Nair, P., et.al. J. Med. Chem. 50, 2779-2786 (2007), http://dx.doi.org/10.1021/im061369n

3. Yamamoto, T., et.al. J. Med. Chem. 51, 6334-6347 (2008), http://dx.doi.org/10.1021/jm800389v

4. Largent-Milnes, T.M., Yamamoto, T., Nair, P., Moulton, J.W., Hruby, V.J., Lai, J., Porreca, F., Vanderah

T.W. Brit. J. Pharmacol. 161, 986-1001 (2010), http://dx.doi.org/10.1111/j.1476-5381.2010.00824.x

5. Largent Milnes, T.M., et.al. J. Pharma. Exp. Therap. 347, 7-19(2013), http://dx.doi.org/10.1124/jpet.113.205245 